\title{
Classifying Recognition Results for Spoken Dialog Systems
}

\author{
Malte Gabsdil \\ Deptartment of Computational Linguistics \\ Saarland University \\ Germany \\ gabsdilecoli.uni-sb.de
}

\begin{abstract}
This paper investigates the correlation between acoustic confidence scores as returned by speech recognizers with recognition quality. We report the results of two machine learning experiments that predict the word error rate of recognition hypotheses and the confidence error rate for individual words within them.
\end{abstract}

\section{Introduction}

Acoustic confidence scores as computed by speech recognizers play an important role in the design of spoken dialog systems. Often, systems solely decide on the basis of an overall acoustic confidence score whether they should accept (consider correct), clarify (ask for confirmation), or reject (prompt for repeat/rephrase) the interpretation of an user utterance. This behavior is usually achieved by setting two fixed confidence thresholds: if the confidence score of an utterance is above the upper threshold it is accepted, when it is below the lower threshold it is rejected, and clarification is initiated in case the confidence score lies in between the two thresholds. The GoDiS spoken dialog system (Larsson and Ericsson, 2002) is an example of such a system. More elaborated and flexible system behavior can be achieved by making use of individual word confidence scores or slot-confidences ${ }^{1}$ that allow more fine-grained de-

\footnotetext{
${ }^{1}$ Some recognition platforms allow the application programmer to associate semantic slot values with certain words of an input utterance. The slot-confidence is then defined as the acoustic confidence for the words that make up this slot.
}

cisions as to which parts of an utterance are not sufficiently well understood.

The aim of this paper is to investigate how well acoustic confidences correlate with recognition quality and to use machine learning (ML) techniques to improve this correlation. In particular, we will conduct two different experiments. First, we try to predict the word error rate (WER) of a recognition result based on its overall confidence score and show that we can improve on this by using ML classifiers. Second, we will consider individual word confidence scores and again show that ML techniques can be fruitfully applied to the task of deciding whether individual words were recognized correctly or not.

The paper is organized as follows. In the next section, we explain the general experimental setup, introduce acoustic confidences, and explain how we labeled our data. Sections 3 and 4 report on the actual experiments. Section 5 summarizes and concludes the paper.

\section{Experimental Setup}

We use the ATIS2 corpus (MADCOW, 1992) as our speech data source. The corpus contains approx. 15.000 utterances and has a vocabulary size of about 1.000 words. In order to get "real" recognition data, we trained and tested the commercial NUANCE8. $0^{2}$ recognition engine on the ATIS 2 corpus. To this end we first split the corpus into two distinct sets. With the first set we trained a statistical language model (trigram) for the recognizer. This model was then

\footnotetext{
${ }^{2}$ http: //www . nuance. com
} 
used to recognize the other set of utterances (using 1-best recognition). Finally, we split the set of recognized utterances into three different sets. A training set $(75 \%)$, a test set $(20 \%)$ and a development set $(5 \%)$.

\subsection{Acoustic Confidences}

The NUANCE recognizer returns an overall acoustic confidence score for each recognition hypothesis as well as individual word confidence scores for each word in the hypothesis. Acoustic confidences are computed in an additional step after the actual recognition process. The aim is to estimate a normalized probability of a (sub-)sequence of words that can be interpreted as a predictor whether the sequence was correctly recognized or not (see (Wessel et al., 2001) for a comparison of different confidence estimators). Acoustic confidence scores are therefore different from the unnormalized scores computed by the standard Viterbi decoding in HMM based recognition which selects the best hypothesis among competing alternatives.

We will use acoustic confidence scores to derive baseline values for the two experiments reported in Sections 3 and 4.

\subsection{Recognition Results}

We first give a general overview of the performance of the NUANCE speech recognizer. Table 1 reports the overall word error rate (WER) in terms of insertions, deletions, and substitutions as computed by the recognition engine (but see the discussion on the Levenstein distance in the next paragraph).

\begin{tabular}{c|c|c|c} 
Insertions & Deletions & Substitutions & WER \\
\hline 1342 & 1693 & 5856 & $\mathbf{1 1 . 8 3}$
\end{tabular}

Table 1: Overall WER

Table 2 shows the absolute number and percentages of the sentences that where recognized correctly (WER0), recognized with a WER between $1 \%$ and 50\% (WER50), and with a WER greater than 50\% (WER100). Rejections and timeouts refer to the number of utterances completely rejected by the recognizer and utterances for which a processing timeout threshold was exceeded. In both cases the recognizer did not return a hypothesis.

\begin{tabular}{l|rr} 
& Abs. & Perc. \\
\hline WER0 & 3824 & $51.0 \%$ \\
WER50 & 3204 & $42.7 \%$ \\
WER100 & 283 & $3.8 \%$ \\
\hline Rejections & 5 & $0.1 \%$ \\
Timeouts & 187 & $2.5 \%$ \\
\hline Total & 7503 & $100.1 \%$
\end{tabular}

Table 2: Recognition results grouped by WER

In our first experiment we will use the three categories WER0, WER50, and WER100 to establish a correlation between the overall acoustic confidence score for an utterance and its word error rate. The basic idea is that these three classes might be used by a system to decide whether it should accept, clarify, or reject an hypothesis.

\subsection{Labeling Words}

We also labeled each word in the set of recognized utterances as either correctly or incorrectly recognized. The labeling is based on the Levenstein distance between the actual transcription of an utterance and its recognition hypothesis. The Levenstein distance computes an alignment that minimizes the number of insertions, deletions, and substitutions when comparing two different sentences. However, this distance can be ambiguous between two or more alignment transcripts (i.e. there are can be several ways to convert one string into another using the minimum number of insertions, deletions, and substitutions). (1) shows two possible alignments for a recognized utterance from the ATIS2 corpus, where ' $m$ ' stands for match, ' $i$ ' for insertion, and ' $s$ ' for substitution.

(1) Ambiguous Levenstein alignment

Trans: are there any stops on that flight

Recog: what are the stops on the flight

Align1: s-s-s-m-m-s-m

Align2: i-m-s-d-m-m-s-m

To avoid this kind of ambiguity, we converted all words to their phoneme representations using the CMU pronunciation dictionary ${ }^{3}$. We then ran

\footnotetext{
${ }^{3}$ http://www. speech.cs.cmu. edu/cgi-bin/ cmudict
} 
the Levenstein distance algorithm on these representations and converted back the result to the word level. This procedure gives us more intuitive alignment results because it has a bias towards substituting phonemically similar words (e.g. Align2 in (1) above). Of course, the Levenstein distance on the phoneme level can again be ambiguous but this is more unlikely since the to-be aligned strings are longer.

We will use the individually labeled words in our second experiment where we try to improve the confidence error rate and the detection-error tradeoff curve for the recognition results.

\section{Experiment 1}

The purpose of the first experiment was to find out how well features that can be automatically derived from a recognition hypothesis can be used to predict its word error rate.

As already mentioned in the previous section, all recognized sentences were assigned to one of the following classes depending on their actual WER: WER0 (WER 0\%, sentence correctly recognized), WER50 (sentences with a WER between $1 \%$ and $50 \%$ ), and WER 100 (sentences with a WER greater than $50 \%$ ). The motivation to split the data into these three classes was that they can be associated with the two fixed thresholds commonly used in spoken dialog systems to decide whether an utterance should be accepted, clarified, or rejected.

We are aware that this might not be an optimal setting. Some spoken dialog systems only spot for keywords or key-phrases in an utterance. For them it does not matter whether "unimportant" words were recognized correctly or not and a WER greater than zero is often acceptable. The main problem is that what counts as a keyword or key-phrase is system and domain depended. We cannot simply base our experiments on the WER for content words like nouns, verbs, and adjectives. In a travel agency application, for example, the prepositions 'to' and 'from' are quite important. In home automation, quantifiers/determiners are important to distinguish between the commands 'switch off all lights' and 'switch off the hall lights' (this example is borrowed from David Milward). For further examples see also (Bos and Oka, 2002).

\subsection{Machine Learners}

We predicted the WER-class for recognized sentences based on their overall confidence score, and with the two machine learners TiMBL (Daelemans et al., 2002) and Ripper (Cohen, 1996). TiMBL is a software package that provides two different memory based learning algorithms, each with finetunable metrics. All our TiMBL experiments were done with the IB 1 algorithm that uses the $k$-nearest neighbor approach to classification: the class of a test item is derived from the training instances that are most similar to it. Memory-based learning is often referred to as "lazy" learning because it explicitly stores all training examples in memory without abstracting away from individual instances in the learning process.

Ripper, on the other hand, implements a "greedy" learning algorithm that tries to find regularities in the training data. It induces rule sets for each class with built-in heuristics to maximize accuracy and coverage. With default settings, rules are first induced for low frequency classes, leaving the most frequent class being the default. We chose TiMBL and Ripper as our two machine learners because they employ different approaches to classification, are wellknown, and widely available.

For all experiments we proceeded as follows: First we used the training set to learn optimal confidence thresholds for the baseline classification and the development set to learn program parameters for the two machine learners, which were then trained on the training set. We then tested these settings on the test set. To be able to statistically compare the results, in a third step, we used the learned program parameters to classify the recognition results in the combined training and test sets in a 10-fold crossvalidation experiment. The optimization and evaluation were always done on the weighted $f_{.5}$-score ${ }^{4}$ for all three classes.

\subsection{Baseline}

As a baseline predictor for class assignment we use the overall confidence score of a recognition result returned by the NUANCE recognizer. To assign the three different classes, we have to learn two confi-

\footnotetext{
${ }^{4} f .5$ is the unbiased harmonic mean of precision $(p)$ and recall $(r): f .5=2 p r /(p+r)$
} 
dence thresholds. Whenever the overall confidence of the recognition result is below the lower threshold, we classify it as WER100, whenever it is above the upper threshold we classify it as WER0, and when it is between we classify it as WER50. We report the weighted $f_{.5}$-score for the test set and the cross-validation experiment as well as the standard deviation for the cross-validation experiment in Table 3 .

\begin{tabular}{l|cc} 
& Weighted $f .5$ & St. Deviation \\
\hline test set & $63.57 \%$ & - \\
crossval & $64.13 \%$ & 1.67
\end{tabular}

Table 3: Baseline results

The confidence scores that maximized the results for the NUANCE recognizer on the test set were 66 and 43 .

\subsection{Classification}

We computed a feature vector representation for each recognition result which served as input for the two machine learners. TiMBL and Ripper. Altogether, 27 features were automatically extracted from the recognizer output and the wave-form files of the individual utterances. These features can be grouped into the following seven different categories.

1. Recognizer Confidences: Overall confidence score, max., min., and range of individual word confidences, descriptive statistics of the individual word confidences

2. Hypothesis Length: Length of audio sample, number of words, syllables, and phonemes (CMU based) in recognition hypothesis

3. Tempo: Length of audio sample divided by the number of words, phones, and syllables

4. Recognizer Statistics: Time needed for decoding

5. Site Information: At which site the speech file was recorded ${ }^{5}$

6. fo Statistics: Mean and max. fo, variance, standard deviation, and number of unvoiced frames $^{6}$

7. RMS Statistics: Mean and max. RMS, variance, standard deviation, number of frames with RMS $<100$
Automatic classification of the recognition results was done with different parameter and feature settings for the machine learners. We hereby coarsely followed (Daelemans and Hoste, 2002) who showed that parameter optimization and feature selection techniques improved classification results with TiMBL and Ripper for a variety of different tasks. First, both learners were run with their default settings. Second, we optimized the parameters for the two learners on the development set. Finally, we used a forward feature selection algorithm interleaved with parameter optimization for TiMBL. This algorithm starts out with zero features, adds one feature, and performs parameter optimization. This is done for all features and the five best results are stored. The algorithm then iterates and adds a second feature to these five best parameter settings. Again, parameter optimization is done for every possible feature combination. The algorithm stops when there is no improvement for either of the five best candidates when adding an additional feature. Keeping the five best parameter settings ensures that the feature selection is not too greedy. If, for example, a single feature gives good results but the combination with other features leads to a drop in performance, there is still a change that, say, the second or third best feature from the previous iteration combines well with a new feature and leads to better results.

We report the results for TiMBL (Table 4) and Ripper (Table 5), respectively.

\begin{tabular}{l|cc} 
& Weighted $f .5$ & St. Deviation \\
\hline & \multicolumn{2}{|c}{ Default Settings } \\
test set & $60.44 \%$ & - \\
crossval & $61.24 \%$ & 1.46 \\
\hline & Parameter & Optimization \\
test set & $68.44 \%$ & - \\
crossval & $68.59 \%$ & 2.03 \\
\hline. & Feature Selection \\
test set & $66.41 \%$ & - \\
crossval & $67.01 \%$ & 2.14
\end{tabular}

Table 4: TiMBL results

\footnotetext{
${ }^{5}$ The ATIS2 data was recorded at several different sites.

${ }^{6}$ The fO and RMS (root mean square; a measure of the signal energy level) features were extracted with Entropic's get_fo tool.
} 


\begin{tabular}{|c|c|c|}
\hline & Weighted $f_{, 5}$ & St. Deviation \\
\hline & \multicolumn{2}{|c|}{ Default Settings } \\
\hline test set & $67.97 \%$ & - \\
\hline \multirow[t]{2}{*}{ crossval } & $68.60 \%$ & 1.54 \\
\hline & Parameter & ptimization \\
\hline test set & $68.11 \%$ & - \\
\hline crossval & $68.23 \%$ & 1.46 \\
\hline
\end{tabular}

Table 5: Ripper results
The results show that TiMBL profits from parameter optimization and feature selection. One reason for this is that, with default settings, TiMBL only considers the nearest neighbor in deciding which class to assign to a test item. In our experiment, considering more than one neighbor lead to a better $f_{.5}$ score for the majority class (WER0) which in turn had an impact on overall weighted $f .5$-score. A surprising finding is that the feature selection algorithm did not lead to an improvement. We expected a better score based on (Daelemans and Hoste, 2002) and because some aspects in the feature vector specification (e.g. tempo) are heavily correlated which can cause problems for memory based learners. However, it turned out that our algorithm stopped after selecting only seven of the 27 features which indicates that it might still be too greedy. Another explanation for the results is that optimization with feature selection can be particularly prone to overfitting: The weighted $f .5$-score for the development data, which we used to select features and optimize parameters, was $77.40 \%$ (almost $11 \%$ better than the performance on the test set).

Parameter optimization did not improve the results for Ripper. Compared to TiMBL the smaller standard deviation in the cross-validation results indicates a more uniform/stable classification of the data.

\subsection{Significance}

We used related t-tests and Wilcoxon signed ranks statistics to compare the cross-validation results. All test were done two-tailed at a significance level of $p=.01$. We found that the results for TiMBL with default settings are significantly worse than all other results. The other four machine learning results (parameter optimization and feature selection for TiMBL as well as defaults and parameter optimization for Ripper) significantly outperform the baseline. We could not find a significant difference between the TiMBL (excluding default settings) and Ripper results. In all comparisons, $t$-test and Wilcoxon signed ranks lead to the same results.

\subsection{Ripper Rule Inspection}

During learning, Ripper generates a set of (human readable) decision rules that indicate which features were most important in the classification process. We cannot give a detailed analysis of the induced rules because of space constraints, but Table 6 provides a simple breakdown by feature groups that shows how often features from each group appeared in the rule set. ${ }^{7}$

1. Recognizer Confidences: 25

2. Hypothesis Length: 12

3. Tempo: 1

4. Recognizer Statistics: 8

5. Site Information: 0

6. fo Statistics: 3

7. RMS Statistics: 2

Table 6: Features used by Ripper

We can see that all feature groups except "Site Information" contribute to the rule set. The single most often used feature was the mean of all individual word confidences ( 9 times), followed by the minimum individual word confidence and recognizer latency (both 8 times). The overall acoustic confidence score appeared in 4 rules only.

\section{Experiment 2}

The aim of the second experiment was to investigate whether we can improve the confidence error rate (CER) for the recognized data. The CER measures how good individual word confidence scores predict whether words are correctly recognized or not. A confidence threshold is set according to which all words are either tagged as correct or incorrect. The

\footnotetext{
${ }^{7}$ The figures reported in Table 6 were obtained by training Ripper on the training set with default parameters. Altogether, 16 classification rules were generated.
} 
CER is then simply defined as the number of incorrectly assigned tags divided by the total number of recognized words. The CER is a very simple measure that strongly depends on the tagging threshold and the prior probability of the classes correct and incorrect. Since we have a strong bias towards correct words in our data, we complement the CER evaluation with a second evaluation matrix, the detection-error tradeoff (DET) curve which plots the false acceptance rate (the number of incorrect words tagged as correct divided by the total number of incorrect words) over the false rejection rate (the number of correct words tagged as incorrect divided by the total number of correct words). This curve is instructive because it shows the results for several different tagging thresholds and how they effect the prediction accuracy for the two classes.

\subsection{Features}

The feature vector for the machine learners in the second experiment consisted of 17 features which were automatically derived from the recognition results and the output of Experiment 1. We can again group them into different categories.

1. Overall Confidence: Overall confidence score of the hypothesis the to-be-classified word appears in

2. Left word context: The two word forms left of the to-be-classified word and their individual word confidence scores

3. Word: The to-be-classified word from, its individual word confidence, and two length measures

4. Right word context: The two word forms left of the to-be-classified word and their individual word confidence scores

5. WER estimate: The WER class as assigned to the sentence the to-be-classified word appears in based on the best results from Experiment 1

6. Sentence Length: Three different length measures for the recognition hypothesis the to-beclassified word appears in

\subsection{Confidence Error Rates}

We report the confidence error rates for the test set and cross-validation on the combined train and test sets in Table 7. The machine learners were only run with their default settings.

\begin{tabular}{l|cc} 
& CER & St. Deviation \\
\hline & \multicolumn{2}{|c}{ Baseline } \\
test set & $11.47 \%$ & - \\
crossval & $11.23 \%$ & 0.67 \\
\hline & \multicolumn{2}{|c}{ TiMBL } \\
test set & $11.44 \%$ & - \\
crossval & $11.30 \%$ & 0.55 \\
\hline & \multicolumn{2}{|c}{ Ripper } \\
test set & $13.17 \%$ & - \\
crossval & $10.82 \%$ & 0.68
\end{tabular}

Table 7: CER results

As in Experiment 1, we used related t-tests and Wilcoxon signed ranks statistics to compare the results. Unfortunately, we could not find a significant improvement for the machine learners as compared to the baseline. Both tests show that there is no significant difference between either of the three results for two tailed tests at $p=.01$. Note, however, that the CER is strongly dependent on the prior probabilities of the classes correct and incorrect. It is therefore interesting to compare the performance on the minority class (incorrect) for the baseline, TiMBL, and Ripper. Table 8 shows precision, recall, and $f .5^{-}$ scores on the test set.

\begin{tabular}{l|lll} 
& prec & recall & $f_{.5}$ \\
\hline baseline & 56.93 & 8.90 & 15.39 \\
TiMBL & 51.79 & 35.54 & 42.15 \\
Ripper & 50.84 & 27.55 & 35.74
\end{tabular}

Table 8: Minority class classification

We can see that the baseline performs very poor on the minority class. Indeed the optimal threshold computed during training was 15 which means that almost every word is tagged as correct. This difference does not show up in the CER because it is "overshadowed" by the majority class. The next paragraph will show the advantage of the machine learners when we give equal weight to both the majority and minority classes.

\subsection{Detection-Error Tradeoff}

We use the data from all words in the training and test sets to plot detection-error tradeoff curves. To 
get the baseline DET curve (based on the individual word confidence computed by the NUANCE recognizer) we simply vary the tagging threshold between 100 and 0 and apply it to the data. A threshold of 50 , for example, will classify all words with a confidence higher or equal than 50 as correct and all others as incorrect. The result is a gradual decline in the false rejection rate: When the threshold is 100 , all instances will be tagged as incorrect, when it is 0 , all instances will be tagged as correct.

\subsection{Training Set Composition}

We classified the same data with the machine learners using several 5-fold cross-validation experiments. One big obstacle with the machine learners was that we wanted to force them to gradually produce more false acceptances and less false rejections. Ripper provides a parameter to change the "loss ratio", i.e the ratio of the cost of a false negative to the cost of a false positive. This is exactly what we want but we found that we cannot linearly vary this parameter in a way that gives us a smooth transition between false acceptances and false rejections.

We solved this problem by conducting experiments were we changed the ratio of examples from the two classes within the training set. This was done as follows. During cross-validation we first set aside an equal number of examples from both classes from the training set. Depending on the ratio value, we then added a certain fraction of one of these two sets to the other set. For example, to get a $50 / 50$ ratio, we simply combined the two sets; for a $75 / 25$ ratio we took the first set and added to it $50 \%$ (randomly selected) items from the second set. This procedure in itself does not ensure a smooth transition from false rejections to false acceptances but it worked very well in practice. Note that we basically only take out a certain number of elements from the cross-validation training set. We do still test every data point since we do not change the ratio within the test sets.

Figures 1 and 2 show the DET curves for TiMBL and Ripper as compared to the baseline respectively.

\subsection{Results}

The DET curve for TiMBL is almost identical to the baseline. The curve for Ripper, however, does im-

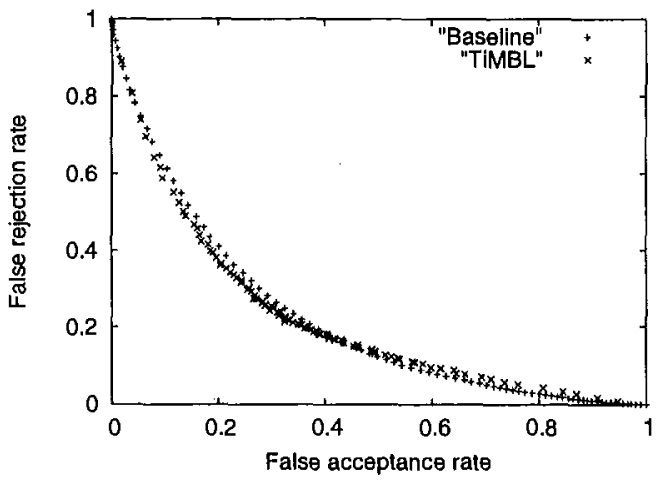

Figure 1: DET for TiMBL

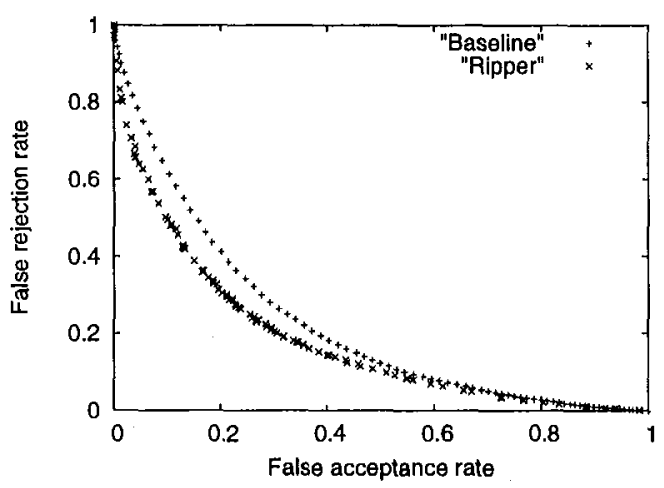

Figure 2: DET for Ripper

prove over the baseline, especially for false acceptance rates (FAR) up to 0.5 . This is an interesting finding because we are often interested in a good performance for the minority class without loosing too much accuracy on the majority class. For spoken dialog systems it is of major importance to be "conservative" and to spot most of the erroneous words in order to avoid misunderstandings. But this is always at the cost of inefficient and annoying dialogs where the system rejects too many utterances or asks too many clarification questions. Figure 2 shows an improvement on the part of the curve where the FAR is low (i.e. where not many erroneous words are accepted). For a FAR of $20 \%$ (i.e. only every fifth incorrect word is not detected as such) Ripper improves the false rejection rate (FRR) by $10 \%$ as compared to the baseline. Figure 2 also shows an improvement in the equal error rate (the point where FAR and FFR are the same) from $28,5 \%$ to $25 \%$. 


\subsection{Ripper Rule Inspection}

Again, we can investigate the rule sets generated by Ripper to find out which features were particularly useful for classification. In Table 9, we report a breakdown by feature groups for one of the crossvalidation folds that lead to a FAR of about $20 \%$ and a FRR of about $30.5 \%$ (i.e. one of the data points that showed the highest improvement over the baseline). The rule set included 15 different rules.

1. Overall Confidence: 7

2. Left word context: 16

3. Word: 21

4. Right word context: 8

5. WER estimate: 2

6. Sentence Length: 3

Table 9: Features used by Ripper

Table 9 shows that features from all six feature groups were used for classification. The single most often used feature was the individual word confidence of the target word (used in all rules), followed by the word confidence of the immediately preceeding word (which appeared in 11 rules).

\section{Conclusions}

Spotting erroneous utterances and words is a major task in spoken dialog systems. Depending on the judgment of recognition quality important decisions are made as to how the dialog should proceed. In this paper, we reported on two experiments that show how machine learning techniques can be used to predict the quality of recognition hypotheses. We both looked at hypotheses as a whole (in terms of their WER) and the individual words within them (in terms of the CER and DET curves). We found that by using the machine learners TiMBL and Ripper we can improve the results in both tasks as compared to predicting recognition quality solely on the basis of the acoustic confidence scores returned by the speech recognizer.

Future work aims in two directions. First we want to try to further improve the results presented in this paper by using better optimization methods for the machine learners (e.g. cross-validation optimization to avoid over-fitting on the development data). Further improvement of the results might also be achieved by considering other features for prediction. For example, we can add the words in the recognition hypothesis as a set-valued feature when using Ripper. We also want to do a more thorough investigation of the rule sets generated by Ripper to find out which features were most important for classification. A long-term goal is to combine the (acoustic) quality prediction with a notion of semantic plausibility in an actual dialog system. In particular, we want to use semantic plausibility to rescore/rerank N-best recognition hypotheses.

\section{Acknowledgments}

We want to thank NUANCE Inc. for making available their recognition software for research purposes.

\section{References}

Johan Bos and Tetsushi Oka. 2002. An Inference-based Approach to Dialogue System Design. In Proceedings of Coling 2002, Taipei.

William W. Cohen. 1996. Learning Trees and Rules with Set-valued Features. In Proceedings of the Thirteenth National Conference on Artificial Intel ligence (AAAI96).

Walter Daelemans and Véronique Hoste. 2002. Evaluation of Machine Learning Methods for Natural Language Processing Tasks. In Proceedings of the Third International Conference on Language Resources and Evaluation (LREC 2002), pages 755-760, Las Palmas, Gran Canaria.

Walter Daelemans, Jakub Zavrel, Ko van der Sloot, and Antal van den Bosch. 2002. TIMBL: Tilburg Memmory Based Learner, version 4.2, Reference Guide. In ILK Technical Report 02-01. Available from http://ilk.kub.nl/downloads/pub/ papers/ilk0201.ps.gz.

Staffan Larsson and Stina Ericsson. 2002. GoDiS - Issue-Based Dialogue Management in a MultiDomain, Multi-Language Dialogue System. In Ronnie Smith, editor, Demonstration Abstracts, ACL-02.

MADCOW. 1992. Multi-Site Data Collection for a Spoken Language Corpus. In Speech and Natural Language Workshop. Morgan Kaufmann.

Frank Wessel, Ralf Schlüter, Klaus Macherey, and Herman Ney. 2001. Confidence Measures for Large Vocabulary Continous Speech Recognition. IEEE Transactions on Speech and Audio Processing, 9(3):288298. 